\title{
Research on Synthesis and Properties of 1,2,3-triazoles Compounds
}

\author{
Ziping Qin ${ }^{1}$ \\ School of Medicine \\ Guangxi University of Science and Technology \\ Liuzhou,Guangxi,545006 China
}

\begin{abstract}
This electronic document is a "live" template. The various components of your paper [title, text, heads, etc.] are already defined on the style sheet, as illustrated by the portions given in this document. 1,2,3- triazoles compounds is important $\mathrm{N}$ - heterocydic compounds, in recent years has been widely used in industrial production, $R \& D$, materials science and other fields. Therefore, the paper take dichlorobenzene and difluorobenzene as raw material use some high-quality sterilization group of triazole fungicide; synthesize a series of triazole compounds. In the synthetic reaction, compounds R1, $R 2, R 3$ have not been reported, their structures were confirmed by 1H-NMR, MS and FT-IR analysis, the properties of compounds synthesized were stable, long-term placement had no change in appearance. At the same time, we study the inhibition ability of fungi. The results showed that: inhibition ability of compound $R 1$ was the strongest on mildew and rots fungi; compound $R 2$ has equal inhibition ability for tebuconazole; the other compounds were showed no obvious bacteriostatic effect.
\end{abstract} method

Keywords- 1,2,3- triazole; bacteriostasis; mildew; synthesis

\section{INTRODUCTION}

1,2,3 - triazole compounds are an important class of heterocyclic compounds, easy to form hydrogen bonds and coordination bond, can interact to form a variety of covalent bond. And has anti-tumor, antibacterial, antiviral and anti convulsion and so on many kinds of biological activity ${ }^{[1-4]}$ because of its special structure. In recent years, with the click chemistry (click chemistry) development, preparation of $1 \mathrm{H}-$ 1,2,3- triazole compounds has become extremely simple. Research shows that 1,4- and 1,5-substituted 1,2,3- triazole compounds are microtubule protease inhibitor effect on microtubule system, is expected to become the new target and effective antitumor drugs. In 2011, Hou [1] reported 1,2,3- click reaction synthesis of three azole compounds based on its antitumor drugs, another new target histone deacetylase has good inhibitory effect, some of the compounds exhibited high activity against leukemia cell lines.

At present, the click reaction mainly using in the classical $1 \mathrm{H}-1,2,3-$ triazole compounds, namely in the monovalent ketone catalyzed, through halides with Terminal Alkynes by sodium azide 1,3- dipolar cycloaddition reaction preparation. But this method still has some limitations, such as catalyst is difficult to recycle, reaction conditions are harsh and solvent is not green, restricted the development of the compounds. In addition, the porous copper is twentieth Century new material system, compared with the pure copper, porous copper has the advantages of large specific surface area and permeability, so it is widely used in catalysis, adsorption and separation of nano material assembly. Porous copper to recycle the Changsha Power aperture yuan new material limited liability company production is $5 \mu \mathrm{m}$ as catalyst, the halogenated hydrocarbons and terminal alkyne and azide cyclization reaction, 1,4- substituted 1,2,3- triazole compounds were synthesized by one pot method, and its structure was confirmed by $1 \mathrm{H}$ NMR, 13CNMR, IR and HRMS were characterized, and its activity in vitro were studied

\section{THE EXPERIMENT AL PART}

\section{A. Apparatusand reagents}

ELISA Kit (size AR): ethyl acetate: chemical company in Shanghai; 1,2- two ethyl chloride, dichloromethane: Chemical Corporation of Shanghai; acetyl chloride, anhydrous methanol, ethanol, chloroacetyl chloride, sodium hydroxide, potassium carbonate, anhydrous Magnesium sulfate, Bian Jisan ethyl ammonium chloride, sodium borohydride, tetrahydrofuran, two chlorobenzene, 1,2,4triazole, sodium chloride, m-Difluorobenzene, pchloridebenzoyl chloride, m-chlorobenzoyl chloride, ortho chloro benzoyl chloride, concentrated hydrochloric acid, petroleum ether, hexane, aluminum chloride, sodium borohydride, three methyl iodide sulfoxide, two dimethyl sulfoxide, pyridine: Sinopharm Chemical Reagent Co., Ltd.

TLC of silica gel (GF254): Qingdao marine chemical factory

Column chromatography silica gel (coarse pore (ZCXII): Qingdao marine chemical factory

Rotary evaporator (RE-52): Shanghai Yarong biochemical instrument factory

Vacuum pump (SHZ-D ( III ) type): Gongyi yingyu Yuhua Instrument Factory

Electronic balance (JA2003N type): Shanghai Precision Scientific Instrument Co., Ltd.

Infrared spectroscopy (Nicolet 360): America Nicolet Company

The superconducting NMR spectrometer (BRUKER M-

300): Thermo Nicolet

Mass spectrometry (1100ESI/MS): Agilent

Electric air blowing drying box (DGX-9073BC-1): ShanghaiFuma Experiment Equipment Co., Ltd. 
Magnetic stirrer (DF-101S type): Gongyi yingyu Yuhua Instrument Factory

Ultrasonic cleaner (KQ250 type): Jiangsu Kunshan ultrasonic instruments Co., Ltd.

The heating sleeve (PTHW): Gongyi City Yuhua Instrument Co., Ltd.

\section{B. The synthetic route of triazole compounds}

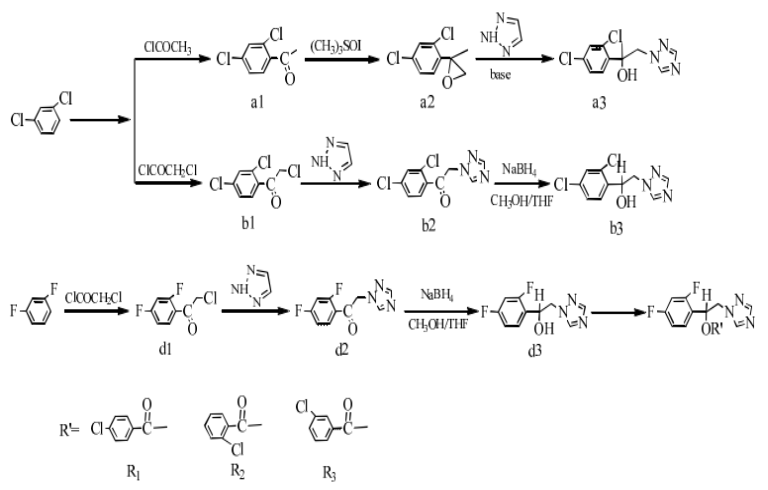

\section{The synthetic methods of triazole compounds}

1) The synthesis of 2,4-dichloroacetophenone (A1)

Equipment of thermometer, constant pressure funnel, reflux condenser (with a drying tube) $250 \mathrm{~mL}$ four mouth flask, add m-chlorobenzene (0.1 mol, $14.7 \mathrm{~g}), \mathrm{AlO} 3(0.17$ mol, $22.7 \mathrm{~g}$ ), the mixture was stirred for $0.5 \mathrm{~h}$ at $30-35{ }^{\circ} \mathrm{C}$, slowly adding acetyl chloride $(0.12 \mathrm{~mol}, 9.42 \mathrm{~g})$, control reaction temperature. After dripping is finished, slowly increasing temperature to 50-55 Deg. C, continue stirring, until there is no hydrogen chloride gas generation, about $5 \mathrm{~h}$ reaction. With the reaction mixture in ice water bath in the flask, the reactant temperature dropped to $5{ }^{\circ} \mathrm{C}$ below, add 3 $\mathrm{mol} / \mathrm{L}$ hydrochloric acid $20 \mathrm{~mL}$ acid solution, the temperature changes little drops of acceleration control mixture. Acid mixture solution after into the beaker, add distilled water, enough to separate solid, negative pressure filtration, drying, to obtain pale yellow solid powder $16.1 \mathrm{~g}$, the yield is $85.2 \%$. Using $n$-hexane recrystallization gets bright yellow crystal.

2,4- dichloroacetophenone (a1): bright yellow crystal, production rate: $85.2 \% .1 \mathrm{H} \mathrm{NMR}(300 \mathrm{MHz}, \mathrm{CDCl} 3, \delta$, ppm, J/Hz): $7.52(1 \mathrm{H}, \mathrm{t}, \mathrm{J}=7.0 \mathrm{~Hz}), 7.38(1 \mathrm{H}, \mathrm{d}, \mathrm{J}=1.8 \mathrm{~Hz})$, 7.32-7.22 (1H, m),2.61 (3H, s); $\mathrm{MS}(\mathrm{m} / \mathrm{z}): 189.0[\mathrm{M}+1]+$, $211.0[\mathrm{M}+\mathrm{Na}]+$.

(2) The synthesis of $\alpha$ - chlorine -2,4Dichloroacetophenone (b1)

Equipment of thermometer, constant pressure funnel, reflux condenser (with a drying tube) $250 \mathrm{~mL}$ four mouth flask, add m-chlorobenzene (0.1 mol, $14.7 \mathrm{~g}), \mathrm{AlCl} 3(0.17$ mol, $22.7 \mathrm{~g}$ ), the mixture was stirred for $0.5 \mathrm{~h}$ at $30-35{ }^{\circ} \mathrm{C}$, slowly dropping chloroacetyl chloride $(0.12 \mathrm{~mol}, 13.56 \mathrm{~g})$, controlling the reaction temperature. After dripping is finished, slowly increasing temperature to 50-55 Deg. C, continue stirring, until there is no hydrogen chloride gas generation, about $5 \mathrm{~h}$ reaction. With the reaction mixture in ice water bath in the flask, the reactant temperature dropped to $5{ }^{\circ} \mathrm{C}$ below, add $3 \mathrm{~mol} / \mathrm{L}$ hydrochloric acid $20 \mathrm{~mL}$ acid solution, the temperature changes little drops of acceleration control mixture. Acid mixture solution after into the beaker, add distilled water, enough to separate solid, negative pressure filtration, drying, to obtain pale yellow solid powder $19.5 \mathrm{~g}$, the yield is $87.4 \%$. Using $\mathrm{n}$-hexane recrystallization acquires bright yellow crystal.

a - chlorine -2,4-Dichloroacetophenone (B1): light yellow crystals, yield: $87.4 \%$. FT-IR $(\mathrm{KBr}$ Pressure plate)v/cm-1: 3428.02, 2946.18, 1700.97, 1627.22, 1582.09, $1548.06, \quad 1468.25, \quad 1398.26,1303.91, \quad 1198.20,1110.79$, 1065.56, 984.52, 872.16, 825.64, 789.86, 723.49, $575.93,467.94 ; 1 \mathrm{H} \mathrm{NMR}(300 \mathrm{MHz}, \mathrm{CDCl} 3, \delta, \mathrm{ppm}, \mathrm{J} / \mathrm{Hz})$ : $7.55(1 \mathrm{H}, \mathrm{m}), 7.47(1 \mathrm{H}, \mathrm{s}), 7.36(1 \mathrm{H}, \mathrm{m}), 4.66(2 \mathrm{H}, \mathrm{s})$; $\mathrm{MS}(\mathrm{m} / \mathrm{z}): 222.9[\mathrm{M}+1]+$.

(3)The synthesis of $\alpha$ - chlorine -2,4Dichloroacetophenone (d1)

Equipment of thermometer, constant pressure funnel, reflux condenser (with a drying tube) $250 \mathrm{~mL}$ four mouth flask, add m-fluorobenzene (0.1 mol, $14.7 \mathrm{~g}), \mathrm{AlCl} 3(0.17$ mol, $22.7 \mathrm{~g}$ ), the mixture was stirred for $0.5 \mathrm{~h}$ at $30-35{ }^{\circ} \mathrm{C}$, slowly dropping chloroacetyl chloride $(0.12 \mathrm{~mol}, 13.56 \mathrm{~g})$, controlling the reaction temperature. After dripping is finished, slowly increasing temperature to 50-55 Deg. C, continue stirring, until there is no hydrogen chloride gas generation, about $5 \mathrm{~h}$ reaction. With the reaction mixture in ice water bath in the flask, the reactant temperature dropped to $5{ }^{\circ} \mathrm{C}$ below, add $3 \mathrm{~mol} / \mathrm{L}$ hydrochloric acid $20 \mathrm{~mL}$ acid solution, the temperature changes little drops of acceleration control mixture. Acid mixture solution after into the beaker, add distilled water, enough to separate solid, negative pressure filtration, drying, to obtain pale yellow solid powder $16.9 \mathrm{~g}$, the yield is $89.3 \%$. The pale yellow solid powder with $\mathrm{n}$-hexane recrystallization gets bright yellow crystal.

a - chlorine -2,4-Dichloroacetophenone (B1): light yellow crystals, yield: $89.3 \%$. FT-IR( $\mathrm{KBr}$ 压片)v/cm- 1 : 3429.12 , 2947.28, 1701.97, 1628.32, 1583.19, 1549.36, $1469.55, \quad 1399.36,1304.96, \quad 1198.50, \quad 1110.89, \quad 1066.76$, $985.62,87326,826.74,789.96,724.49,576.93,468.97 ; 1 \mathrm{H}$ NMR (300 MHz, CDCl3, $\delta, \mathrm{ppm}, \mathrm{J} / \mathrm{Hz}): 8.36(1 \mathrm{H}, \mathrm{m}), 7.56$ $(1 \mathrm{H}, \mathrm{s}), 7.47(1 \mathrm{H}, \mathrm{m}), 4.75(2 \mathrm{H}, \mathrm{s}) ; \mathrm{MS}(\mathrm{m} / \mathrm{z}): 189.99[\mathrm{M}+1]+$.

(4) M-CHLOROBENZOIC acid 1-(2,4- dienide)-2-(1H1,2,4- riazole -1-baseed)- ethyl (R3) In a constant pressure drop funnel four mouth flask, add D3 (0.02 mol, $4.5 \mathrm{~g})$, pyridine $(10 \mathrm{~mL})$, stirring, slowly dropping $\mathrm{m}$-chlorobenzoyl chloride $(0.022 \mathrm{~mol}, 3.85 \mathrm{~g})$, dropping after $3 \mathrm{~h}$, reaction at room temperature. In 5\% hydrochloric acid washing 3 times, then the organic layer, washing the organic phase to neutral, rotary evaporation, methanol washing, drying to obtain solid white $6.2 \mathrm{~g}$, the yield is $85.3 \%$.

M-CHLOROBENZOIC acid 1-(2,4- dienide)-2- $(1 \mathrm{H}-$ 1,2,4- riazole -1-baseed)- ethyl (R3) : white solid, yield:85.3 \%. FT-IR(KBr) v/cm-1: 3417.59, 3124.84, $3114.66, \quad 3075.73,1719.69, \quad 1622.79, \quad 1507.03,1605.95$, $1575.22, \quad 1448.26, \quad 1428.26, \quad 1375.78, \quad 1363.25,1355.20$, $1326.02,1303.79,1287.60,1272.34,1253.08,1214.37$, $1117.83,1090.78,1071.90,1035.00,1003.44,966.12,899.28$, $872.51,751.68,739.36,676.82,669.70,659.26,647.65$, $607.89,530.26,507.36,486.02 ; 1 \mathrm{H}$ NMR (300 MHz, CDCl3, 
$\delta, \mathrm{ppm}, \mathrm{J} / \mathrm{Hz}): 8.06(1 \mathrm{H}, \mathrm{s}), 7.99(1 \mathrm{H}, \mathrm{s}), 7.91(2 \mathrm{H}, \mathrm{m}), 7.57$ $(1 \mathrm{H}, \mathrm{m}), 7.41(1 \mathrm{H}, \mathrm{m}), 7.34-7.21(1 \mathrm{H}, \mathrm{m}), 6.89(2 \mathrm{H}, \mathrm{m})$, $6.56(1 \mathrm{H}, \mathrm{m}), \quad 4.75(2 \mathrm{H}, \mathrm{m}) ; \quad \mathrm{MS}(\mathrm{m} / \mathrm{z}): 364.1[\mathrm{M}+1]+$, $386.1[\mathrm{M}+\mathrm{Na}]+$.

\section{THE RESULTS ANALYSIS OF 1H-NMR COMPOUNDS}

The 1H-NMR analysis results see Table 1, the spectrum analysis results show that the target compounds A1-A3, b1b3, D1-D3, R1-R3 structure and the expected structure.

Table $1{ }^{1} \mathrm{H}-\mathrm{NMR}$ and MS data of the target compounds

\begin{tabular}{|c|c|}
\hline $\begin{array}{l}\text { Co } \\
\text { mpd. }\end{array}$ & 1H-NMR and MS \\
\hline a1 & $\begin{array}{l}\delta: 7.52(1 \mathrm{H}, \mathrm{t}, \mathrm{J}=7.0 \mathrm{~Hz}), 7.38(1 \mathrm{H}, \mathrm{d}, \mathrm{J}= \\
1.8 \mathrm{~Hz}), \quad 7.32-7.22 \quad(1 \mathrm{H}, \quad \mathrm{m}), \quad 2.61 \quad(3 \mathrm{H}, \mathrm{s}) \\
\mathrm{MS}(\mathrm{m} / \mathrm{z}): 189.0[\mathrm{M}+1]+, 211.0[\mathrm{M}+\mathrm{Na}]+.\end{array}$ \\
\hline $\mathrm{a} 2$ & $\begin{array}{l}\delta: 7.41(1 \mathrm{H}, \mathrm{d}, \mathrm{J}=8.3 \mathrm{~Hz}), 7.33(1 \mathrm{H}, \mathrm{t}, \mathrm{J}= \\
4.2 \mathrm{~Hz}), 7.23(1 \mathrm{H}, \mathrm{dd}, \mathrm{J}=8.3,2.0 \mathrm{~Hz}), 2.98-2.79 \\
(2 \mathrm{H}, \mathrm{m}), 1.69(3 \mathrm{H}, \mathrm{d}, \mathrm{J}=61.0 \mathrm{~Hz}) ; \mathrm{MS}(\mathrm{m} / \mathrm{z}) \\
204.1[\mathrm{M}+1]+, 226.1[\mathrm{M}+\mathrm{Na}]+.\end{array}$ \\
\hline a3 & $\begin{array}{l}\delta: 7.96(1 \mathrm{H}, \mathrm{s}), 7.81(1 \mathrm{H}, \mathrm{s}), 7.66(1 \mathrm{H}, \mathrm{t}, \mathrm{J}= \\
7.1 \mathrm{~Hz}), 7.33(1 \mathrm{H}, \mathrm{d}, \mathrm{J}=2.1 \mathrm{~Hz}), 7.16(1 \mathrm{H}, \mathrm{dd}, \mathrm{J}= \\
8.6,2.2 \mathrm{~Hz},), 5.15-4.54(2 \mathrm{H}, \mathrm{m}), 1.70-4.50(1 \mathrm{H}, \\
\text { s), } 1.68 \quad(3 \mathrm{H}, \mathrm{s}) ; \mathrm{MS}(\mathrm{m} / \mathrm{z}): 272.1 \quad[\mathrm{M}+1]+, \\
294.1[\mathrm{M}+\mathrm{Na}]+.\end{array}$ \\
\hline b1 & $\begin{array}{c}\delta: 7.55(1 \mathrm{H}, \mathrm{m}), 7.47(1 \mathrm{H}, \mathrm{s}), 7.36(1 \mathrm{H}, \mathrm{m}), \\
4.66(2 \mathrm{H}, \mathrm{s}) ; \mathrm{MS}(\mathrm{m} / \mathrm{z}): \mathrm{m} / \mathrm{z} 222.9[\mathrm{M}+1]+.\end{array}$ \\
\hline b2 & $\begin{array}{l}\quad \delta: \quad 8.24(1 \mathrm{H}, \mathrm{s}), 7.98(1 \mathrm{H}, \mathrm{s}), 7.65(1 \mathrm{H}, \mathrm{d}, \mathrm{J}= \\
8.4 \mathrm{~Hz}), 7.51(1 \mathrm{H}, \mathrm{d}, \mathrm{J}=1.8 \mathrm{~Hz}), 7.40(1 \mathrm{H}, \mathrm{m}), \\
5.62 \quad(2 \mathrm{H}, \quad \mathrm{s}) ; \quad \mathrm{MS}(\mathrm{m} / \mathrm{z}): 256.0 \quad[\mathrm{M}+1]+, \\
278.0[\mathrm{M}+\mathrm{Na}]+.\end{array}$ \\
\hline b3 & $\begin{array}{l}\delta: \quad 8.13(1 \mathrm{H}, \mathrm{s}), 7.78(1 \mathrm{H}, \mathrm{s}), 7.51-7.46(1 \mathrm{H}, \\
\mathrm{m}), \quad 7.38(1 \mathrm{H}, \mathrm{m}), 7.28-7.25(1 \mathrm{H}, \mathrm{m}), 5.39-5.36 \\
(1 \mathrm{H}, \mathrm{m}), 4.69-3.97(2 \mathrm{H}, \mathrm{m}), 2.02-1.22(1 \mathrm{H}, \mathrm{m}) \\
\mathrm{MS}(\mathrm{m} / \mathrm{z}): 258.1[\mathrm{M}+1]+, 280.1[\mathrm{M}+\mathrm{Na}]+.\end{array}$ \\
\hline d1 & $\begin{array}{l}\delta: 8.36(1 \mathrm{H}, \mathrm{m}), 7.56(1 \mathrm{H}, \mathrm{s}), 7.47(1 \mathrm{H}, \mathrm{m}), \\
4.75(2 \mathrm{H}, \mathrm{s}) ; \mathrm{MS}(\mathrm{m} / \mathrm{z}): 189.99[\mathrm{M}+1]+.\end{array}$ \\
\hline $\mathrm{d} 2$ & $\begin{array}{l}\delta: 8.22(1 \mathrm{H}, \mathrm{s}), 8.08-8.00(2 \mathrm{H}, \mathrm{dd}, \mathrm{J}=15.1, \\
8.5 \mathrm{~Hz}), 7.08-6.94(2 \mathrm{H}, \mathrm{m}), 5.59(2 \mathrm{H}, \mathrm{d}, \mathrm{J}=3.3 \\
\mathrm{Hz}) ; \mathrm{MS}(\mathrm{m} / \mathrm{z}): 224.1[\mathrm{M}+1]+, 246.1[\mathrm{M}+\mathrm{Na}]+.\end{array}$ \\
\hline d3 & $\begin{array}{l}\delta: \quad 8.02(1 \mathrm{H}, \mathrm{s}), 7.83(1 \mathrm{H}, \mathrm{s}), 7.54-7.34(1 \mathrm{H}, \\
\mathrm{m}), \quad 6.96-6.78 \quad(2 \mathrm{H}, \mathrm{m}), 5.34 \quad(1 \mathrm{H}, \mathrm{m}), 4.55- \\
4.18(2 \mathrm{H}, \mathrm{m}), 3.00-1.56(1 \mathrm{H}, \mathrm{m}) ; \mathrm{MS}(\mathrm{m} / \mathrm{z}): 226.1 \\
{[\mathrm{M}+1]+, 248.1[\mathrm{M}+\mathrm{Na}]+.}\end{array}$ \\
\hline r1 & $\begin{array}{l}\delta: \quad 8.02(1 \mathrm{H}, \mathrm{s}), 7.94-7.90(2 \mathrm{H}, \mathrm{m},), \\
7.88(1 \mathrm{H}, \mathrm{s}), 7.44-7.35(2 \mathrm{H}, \mathrm{m}), 7.29-7.19(1 \mathrm{H}, \\
\mathrm{m}), 6.90-6.79(2 \mathrm{H}, \mathrm{m}), 6.56-6.48(1 \mathrm{H}, \mathrm{m}), 4.83- \\
4.57 \quad(2 \mathrm{H}, \mathrm{m}) ; \quad \mathrm{MS}(\mathrm{m} / \mathrm{z}): 364.1 \quad[\mathrm{M}+1]+, \\
386.1[\mathrm{M}+\mathrm{Na}]+.\end{array}$ \\
\hline r2 & $\begin{array}{l}\delta: \quad 8.08(1 \mathrm{H}, \mathrm{s}), 7.91(1 \mathrm{H}, \mathrm{s}), 7.80-7.78(1 \mathrm{H}, \\
\mathrm{s}), 7.46-7.44(2 \mathrm{H}, \mathrm{m}), 7.35-7.30(2 \mathrm{H}, \mathrm{m}), 6.85- \\
6.90(2 \mathrm{H}, \mathrm{m}), 6.59-6.56(2 \mathrm{H}, \mathrm{m}), 4.78-4.67(1 \mathrm{H}, \\
\mathrm{m}) ; \mathrm{MS}(\mathrm{m} / \mathrm{z}): 364.1[\mathrm{M}+1]+.\end{array}$ \\
\hline r3 & $\begin{array}{l}\delta: 8.06(1 \mathrm{H}, \mathrm{s}), 7.99(1 \mathrm{H}, \mathrm{s}), 7.91(2 \mathrm{H}, \mathrm{m}), \\
7.57(1 \mathrm{H}, \mathrm{m}), 7.41(1 \mathrm{H}, \mathrm{m}), 7.34-7.21(1 \mathrm{H}, \mathrm{m}), \\
6.89(2 \mathrm{H}, \mathrm{m}), \quad 6.56(1 \mathrm{H}, \mathrm{m}), \quad 4.75(2 \mathrm{H}, \mathrm{m}) \\
\mathrm{MS}(\mathrm{m} / \mathrm{z}): 364.1[\mathrm{M}+1]+, 386.1[\mathrm{M}+\mathrm{Na}]+.\end{array}$ \\
\hline
\end{tabular}

\section{ANALYSIS OF THE ANTIMICROBIAL ACTIVITY FOR COMPOUNDS}

This paper take diffusion method to detect antiseptic antibacterial effect of target compounds, and has been used as a wood preservative tebuconazole as control agents, compare them to four kinds of decay fungi (Coriolus versicolor, brown rot fungi, Aspergillus, Trichoderma viride) antibacterial activity.

In the same concentration, the inhibition zone diameter, antibacterial effect of compound R1 on four kinds of decay fungi obviously; while the compound D3 had a slight inhibitory effect on Aspergillus niger, the other three strains showed no antibacterial effect; equivalent inhibitory effect of compound A3, B3, R3 to four kinds of bacteria all, bacteriostatic effect than control fungicides tebuconazole difference; antibacterial effect and tebuconazole quite compound R2. With the increase of drug concentration, R1, $\mathrm{R} 2$ and compound control medicament tebuconazole were increased; for compounds A3, B3, R3, and only when the concentration reaches a certain value showed inhibitory effect, its antibacterial ability was enhanced with the concentration increase of the concentration of D3 reached compounds; the maximum value of $2 \%$, of Coriolus versicolor, gloeophyllum trabeum and Trichoderma viride showed no inhibitory effect, when the concentration was $1 \%$, showing slight inhibiting effect on Kuroma.

\section{CONCLUSIONS}

1, 2, 3- triazole compounds and their derivatives have been widely used as nitrogen heterocyclic five membered ring compounds, in pesticide and medic ine, poly mer, surface chemistry field have extensive application. Difluoromethyl $(\mathrm{CF} 2 \mathrm{H})$, trifluoromethyl $(\mathrm{CF} 3)$ because of its special physical and chemical properties, it will be introduced to the small organic molecules can often affect the bioactivity of compounds, thus in drug design and development of pesticides is attracting more and more attention. Multi component reactions can be quickly synthesized a series of diverse compounds and complex and the establishment of corresponding compound library, in recent years has been rapid development in material science, medicine, pesticide and natural products and their derivatives. We believe that, with the 1,2,3- thiazole synthesis method of continuous improvement, click chemical will be extensively applied in drug research, bio conjugation, material synthes is and so on.

\section{REFERENCE}

[1]FU X, ALBERMANN C, ZHANG C,et al. Diversifying vancomycin via chemoenzymatic strategies[J].OrgLett, 2005, 7(8):1513-1515.

[2]WANG X L, WAN K, ZHOU C H. Synthesis of novel sulfanilamidederived 1, 2, 3-triazoles and their evaluation for antibacterial and antifungal activities[J].Eur JMed Chem, 2010, 45(8):4631-4639.

[3]DAMODIRANM, MURALIDHARAN D, PERUMAL $P \quad T$. Regioselective synthesis and biological evaluation of bis( indolyl) methane derivatized 1, 4-disubstit uted 1, 2, 3-bistriazoles as antiinfect ive agents[ J].Bioorg Med Chem Lett, 2009, 19 (13):361 1-3614.

[4]LUESSE S B, WELLSG, NAYEK A,etal. Natural products inparallel synthesis: triazole libraries of nonactic acid[J].Bioorg Med Chem Lett, 2008, 18(14): 3946-3949. 
[5]BEENA, KUMAR N, ROHILLA R K,etal. Synthesis and antibacterial activity evaluation of metronidazole-triazole conjugates[J].BioorgMed Chem Lett, 2009, 19(5): 1396-1398.
[6]AHER NG, POREV S, MISHRANN,etal. Synthesis and antifungal activity of1, 2, 3-triazole containing fluconazole analogues[J].BioorgMed Chem Lett, 2009, 19(3): 759-763. 